\title{
SmoCuDa: A Validated Smoking Cue Database to Reliably Induce Craving in Tobacco Use Disorder
}

\author{
Andrei Manoliu a, b, c Amelie Haugg ${ }^{a, d}$ Ronald Sladky a, e, ${ }^{a}$ Lea Hulka ${ }^{a}$ \\ Matthias Kirschner ${ }^{a, g} \quad$ Annette B. Brühl ${ }^{a}{ }^{h} \quad$ Erich Seifritz $^{a} \quad$ Boris Quednow $^{a}$ \\ Marcus Herdener ${ }^{a}$ Frank Scharnowski ${ }^{a}, \mathrm{~d}, \mathrm{e}, \mathrm{i}$ \\ aDepartment of Psychiatry, Psychotherapy and Psychosomatics, Psychiatric Hospital, University of Zurich, Zurich, \\ Switzerland; ' Wellcome Centre for Human Neuroimaging, University College London, London, UK; ${ }^{c}$ Max Planck \\ University College London Centre for Computational Psychiatry and Ageing Research, London, UK; ${ }^{\mathrm{d} N e u r o s c i e n c e}$ \\ Center Zurich, University of Zurich and Swiss Federal Institute of Technology, Zurich, Switzerland; 'Department of \\ Basic Psychological Research and Research Methods, Faculty of Psychology, University of Vienna, Vienna, Austria; \\ fDepartment of Cognition, Emotion, and Methods in Psychology, Faculty of Psychology, University of Vienna,

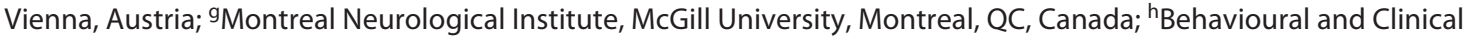 \\ Neuroscience Institute and Department of Psychiatry, University of Cambridge, Cambridge, UK; 'Zurich Center for \\ Integrative Human Physiology (ZIHP), University of Zurich, Zurich, Switzerland
}

\section{Keywords}

Addiction $\cdot$ Cue exposure $\cdot$ Database $\cdot$ Nicotine $\cdot$ Smoking $\cdot$ Tobacco

\begin{abstract}
Background: Cue-reactivity paradigms provide valuable insights into the underlying mechanisms of nicotine craving in nicotine-dependent subjects. In order to study cue-driven nicotine craving, robust and validated stimulus datasets are essential. Objectives: The aim of this study was to generate and validate a large set of individually rated smoking-related cues that allow for assessment of different stimulus intensities along the dimensions craving, valence, and arousal. Methods: The image database consisted of 330 visual cues. Two hundred fifty smoking-associated pictures (Creative Commons license) were chosen from online databases and showed a widespread variety of smoking-associated content. Eighty pictures from previously published databases
\end{abstract}

karger@karger.com www.karger.com/ear

Karger $\stackrel{\text { ' }}{5}$

GOPEN ACCESS
(C) 2020 The Author(s)

Published by S. Karger AG, Basel

This is an Open Access article licensed under the Creative Commons Attribution-NonCommercial-4.0 International License (CC BY-NC) (http://www.karger.com/Services/OpenAccessLicense), applicable to the online version of the article only. Usage and distribution for commercial purposes requires written permission. were included for cross-validation. Forty volunteers with tobacco use disorder rated "urge-to-smoke," "valence," and "arousal" for all images on a 100-point visual analogue scale. Pictures were also labelled according to 18 categories such as lit/unlit cigarettes in mouth, cigarette end, and cigarette in ashtray. Results: Ratings (mean \pm SD) were as follows: urge to smoke, $44.9 \pm 13.2$; valence, $51.2 \pm 7.6$; and arousal, $54.6 \pm$ 7.1. All ratings, particularly "urge to smoke," were widely distributed along the whole scale spectrum. Conclusions: We present a novel image library of well-described smoking-related cues, which were rated on a continuous scale along the dimensions craving, valence, and arousal that accounts for inter-individual differences. The rating software, image database, and their ratings are publicly available at https:// smocuda.github.io.

(c) 2020 The Author(s). Published by S. Karger AG, Basel
Andrei Manoliu

Department of Psychiatry, Psychotherapy and Psychosomatics Psychiatric Hospital, University of Zurich, Lenggstrasse 31 $\mathrm{CH}-8053$ Zurich (Switzerland) andrei.manoliu@bli.uzh.ch 


\section{Introduction}

With over 7 million smoking-related deaths per year [1], tobacco use represents the primary avoidable cause of morbidity and mortality worldwide [2]. In the USA, it is directly related to approximately one-fifth of all deaths [3]. With respect to potential future development, the World Health Organization (WHO) reported that cigarette smoking might lead to 1 billion deaths in the 21st century [4]. In addition, smoking causes healthcare costs of over $\$ 100$ billion as well as $\$ 97$ billion costs related to lost productivity in the USA each year. Therefore, smoking does not only have a tremendous impact on health but also causes great socio-economic costs. However, despite considerable efforts in prevention and treatment of tobacco use disorder (TUD), relapse rates still remain high when smokers attempt to quit [5]. Even with modern treatment approaches, such as a combination of cognitive behavioural therapy and medication, the most common outcome at 1 year following a quit attempt is still "relapse" [6].

Therefore, it is necessary to improve current treatment methods and to develop new treatment approaches that can support smokers in their smoking cessation goals. To do so, a good understanding of the behavioural as well as neural dynamics underlying nicotine dependence is essential. One commonly used approach to study nicotine craving in humans is cue-reactivity paradigms, where subjects are exposed to smoking-associated stimuli and their behavioural or neuronal responses are observed [7, 8]. Cue-reactivity paradigms not only provide valuable insights into craving-related behaviour and into the neural underpinnings of cue-induced nicotine craving, but also can help to identify individualized targets for smoking cessation treatments, for instance, with neurofeedback training $[9,10]$.

In order to successfully design, evaluate, and conduct experiments and/or treatments that aim to investigate or reduce cue-induced nicotine craving, representative and robust naturalistic datasets of smoking cues are essential. For use in studies and future treatments, these datasets should not only provide validated information on the intensity of craving that is induced by the corresponding cues, but also information on other stimulus dimensions, such as valence and arousal, which have also been associated with nicotine dependence [11]. Preferably, in these datasets, the 3 stimulus dimensions craving, valence, and arousal will cover a wide range of different intensities to represent a wide variety of real-life situations. Additionally, a large continuous scale of stimulus dimension in- tensities allows for a more precise investigation of the neurobiological and behavioural underpinnings of cueinduced nicotine craving. For instance, in a clinical research setting, these continuous scales can be used to conduct dynamic shaping experiments, that is, experiments, where the intensity of the images, or the difficulty of the task, is altered based on current task performance [12]. Furthermore, a broader distribution across a wide range of stimuli may enhance the detection of linear and more complex non-linear associations between perceived stimuli and neuronal response. Finally, a subject's preference for specific images is also highly dependent on familiarity of the content and on current fashion and lifestyle trends. Therefore, datasets should also put a strong focus on modern and contemporary images to complement existent datasets with images that were either taken more than 20 years ago [13] or not rated in terms of actual craving [14].

Here, we introduce a novel smoking-related cue database $(\mathrm{SmoCuDa})$ that provides individually rated smoking-related stimuli for assessing smoking cue-induced behavioural, as well as physiological changes in TUD. The nicotine cues cover a wide range of different stimulus intensities along the stimulus dimensions "craving," "arousal," and "valence." Cue ratings of 40 smokers allow for a controlled cue selection and, in particular, can be used for parametric analyses and the development of experimental paradigms along a continuum of stimulus intensities.

Most importantly, to promote open science and to provide novel tools for the study of TUD, the rating tool and the database presented in the current article are publicly available at https://smocuda.github.io.

\section{Methods}

This study was conducted in accordance with the Declaration of Helsinki and was approved by the local ethics committee (University of Zurich). Written informed consent was obtained from all participants prior to inclusion in the study. All participants received monetary compensation for their participation.

\section{Participants}

Forty volunteers with TUD (age $=26.12 \pm 5.74$ years, female: male $=21: 19$, cigarettes $/$ day $=12.58 \pm 5.84$, smoking duration $=7.2 \pm 4.66$ years) participated in the study and rated the cues. One out of the 40 individuals additionally underwent an fMRI scanning session. All participants were recruited from the community using an online advertising platform of the University of Zurich. Inclusion criteria were TUD according to DSM-5 [15]. Exclusion criteria were current or past mental disorders (except TUD), neurological disorders, cardiovascular diseases, pulmonary disease, haematological, endocrine, or major genitourinary disease 
and current intake of any medication (except oral contraceptives) as well as general exclusion criteria for MRI scans, such as metal implants. We also excluded participants who currently or previously used nicotine replacement or other treatments for TUD such as varenicline or bupropion. Past illicit drug use and caffeine consumption were not exclusion criteria, as long as individuals did not meet the criteria for the corresponding substance use disorders according to DSM-5 [15].

\section{Smoking Cues}

In this study, 330 smoking cues were assessed. Two hundred fifty smoking-associated pictures (which establish the SmoCuDa database and were limited to cigarette smoking) were chosen from online databases providing images published with a creative commons license. Those online databases included "flickr" ( $n=148$ images; https://www.flickr.com), "Pixabay" ( $n=70$ images; https://pixabay.com), "morguefile" $(n=26$ images; https:// morguefile.com/), "Unsplash" ( $n=4$ images; https://unsplash. com/), and "StockSnap" ( $n=2$ images; https://stocksnap.io) using the search terms "cigarette*” OR "nicotine*” OR "tobacco" OR "smoking." All images showed a widespread variety of smokingassociated content, such as persons lighting and/or smoking cigarettes in social situations or alone, packages of cigarettes, cigarette vending machines, cigarettes, cigarette butts, and empty or full ashtrays. Two images depicted cigars. Images in black and white were excluded. Pictures were qualitatively assigned to distinct picture categories (such as "lit cigarette in hand" or "pack of cigarettes," see also online suppl. Table 2; for all online suppl. material, see www.karger.com/doi/10.1159/000509758; Fig. 2) by 1 author. Another author checked the assignment of each image to a respective group. Whenever discrepancies between the 2 authors occurred, the assignment was performed in consensus. For purposes of cross-validation, 20 smoking cues and 20 neutral cues from the International Smoking Image Series (ISIS) [13] and 40 neutral cues from the International Affective Picture System (IAPS) [16] were selected, resulting in a total of 330 cues. To ensure a standardized image quality and to optimize all images for the subsequent experiments, all images were resized to $800 \times 600$ pixel using XnConvert (https://www.xnview.com/de/xnconvert/).

\section{Experimental Procedure and Cue Rating}

All 40 participants were instructed to abstain from smoking $1 \mathrm{~h}$ prior to the experiment. Upon arrival, participants' TUD as well as relevant associated parameters were assessed using paperand-pencil questionnaires. In particular, we asked participants to fill out a semi-structured drug assessment interview, which has been previously described in more detail [17], as well as the Fagerström Test for Nicotine Dependence [18], the Cigarette Withdrawal Scale (CWS-21) [19], the Minnesota Nicotine Withdrawal Scale (MNWS) [20], and the Brief Questionnaire of Smoking Urges (QSU) [21]. Rating of the presented cues took place in a dedicated examination room under identical circumstances (identical interior, light setting, and positioning [including using the same table/chair and ensuring the same line of sight]). Cue presentation and corresponding ratings were performed using a custom-made graphical interface using PsychoPy (http://www.psychopy.org). All stimuli were presented at the identical location using identical display settings and had the same resolution $(800 \times 600$ pixel $)$ as well as size $(20 \times 15 \mathrm{~cm})$. To take inter-individual height differ- ences into account and to maximize reactions to pictures [22], participants were allowed to relocate the display according to their ergonomic needs with respect to the position and angle of the display. The procedure of the cue rating was as follows: first, each participant received an instruction to rate each displayed image with respect to "urge-to-smoke," "valence" and "arousal." Specifically, participants were asked to rate their urge to smoke when viewing the presented image from "no urge to smoke" to "very strong urge to smoke," their emotional valence when viewing the presented image from "very unhappy" to "very happy," and their arousal when viewing the presented image from "very aroused" to "very relaxed." All instructions were given in German.

Subsequently, all 330 cues were presented in a randomized order. For each cue, participants rated "urge-to-smoke," "valence," and "arousal" on a 100-point visual analogue scale of $16 \mathrm{~cm}$ length located $2.5 \mathrm{~cm}$ below the displayed cue using a mouse. No time limits were applied with respect to the rating process. The subsequent cue was presented after the last dimension was rated for each cue, respectively. Sessions ended automatically after all ratings were performed.

\section{Statistical Analysis of Image Ratings}

All statistical analyses of image ratings were performed using SPSS 25.0 (SPSS Inc., Chicago, IL, USA). Normal distribution of ratings for "urge to smoke," "arousal," and "valence" was evaluated using the Shapiro-Wilk test for the SmoCuDa database $(n=10,000$ ratings) and all ratings derived from cross-validation $(n=1,600$ and $n=800$ ratings, respectively). Spearman's correlations were used to assess potential correlations between all 3 rating dimensions for all databases. Separate mixed-design ANOVA was used to determine potential interactions between ratings and cue categories (see online suppl. Table 2 for a detailed description of all cue categories) within the SmoCuDa database. To evaluate potential differences in ratings with respect to gender or nicotine dependence, we performed independent $t$ tests. All statistical tests were Bonferroni-corrected for multiple comparisons (corrected significance level $\alpha=0.05$ ).

\section{Results}

\section{The SmoCuDa}

Across all 40 smokers and across all 250 cues from the SmoCuDa database (resulting in 10,000 ratings for each dimension, respectively), descriptive statistics were as follows: urge to smoke, mean $\pm \mathrm{SD}=46.75 \pm 27.27$; arousal, mean $\pm \mathrm{SD}=55.18 \pm 21.69$; and valence, mean $\pm \mathrm{SD}=50.29 \pm 19.86$. Regarding cue-specific ratings, the detailed mean values for all cues with respect to urge to smoke, valence, and arousal are presented in online suppl. Table $1 \mathrm{~A}$ and presented as raincloud plots in Figure 1. Since fatigue-induced effects have been reported when rating a large number of cues (23), the descriptive analysis was repeated for each volunteer's first 100 presented images (see online suppl. Table 1B; Fig. 2) and compared to values derived from the descriptive analysis based on 

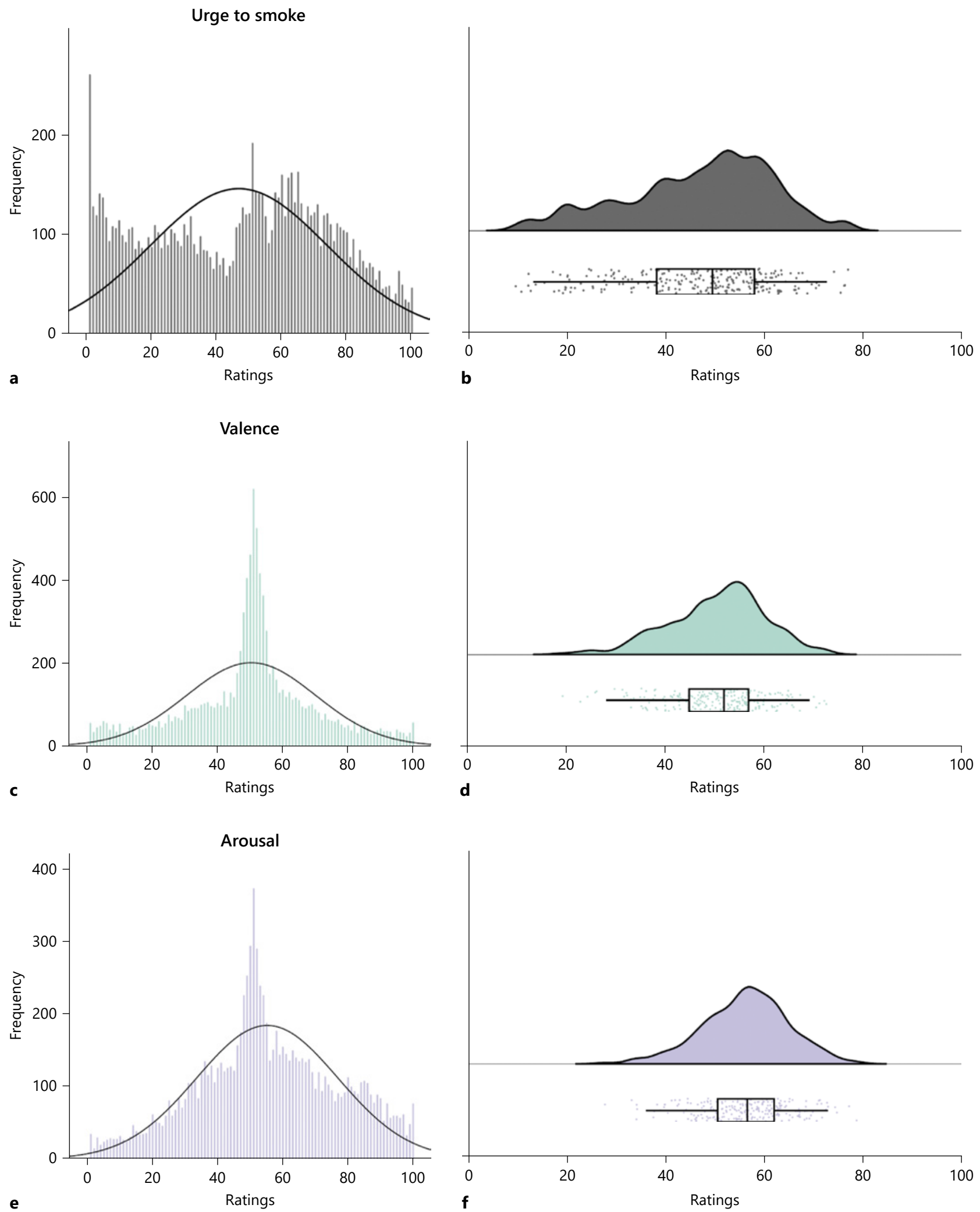

1

(For legend see next page.) 
Fig. 2. Impact of image category on ratings. Average ratings ( $y$-axis) are presented for urge to smoke (grey), arousal (purple), and valence (turquoise) for all 18 image categories. Image categories are ordered according to craving rating. Number of images per category is indicated in parentheses. See also online suppl. Table 3 for detailed presentation of corresponding statistics.
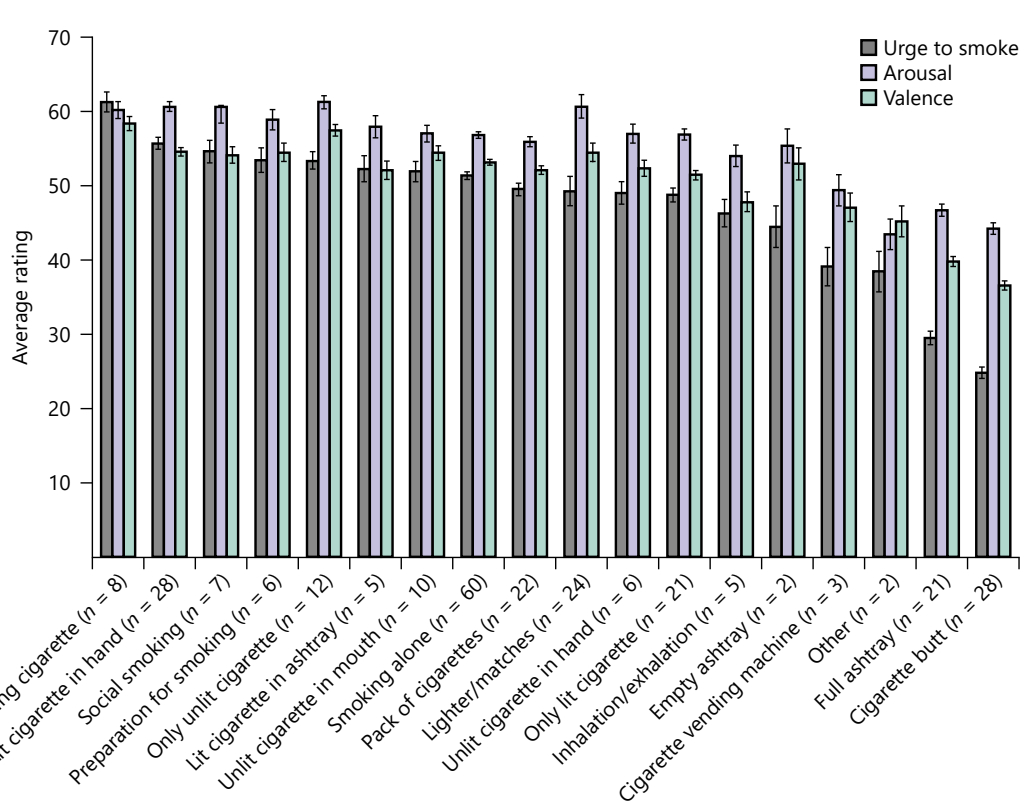

all performed ratings. Average ratings based on the first 100 presented images correlated highly with ratings based on all presented images (urge to smoke: rho $=$ $0.880, p<0.001$; arousal: rho $=0.778, p<0.001$; valence: rho $=0.871, p<0.001)$. Ratings for urge to smoke, arousal, and valence were not normally distributed $(p<0.05$, corrected for multiple comparisons). All ratings correlated significantly with each other (online suppl. Fig. 1; urge to smoke and arousal: rho $=0.314$, urge to smoke and valence: rho $=0.548$, arousal and valence: rho $=$ 0.510 ; all correlations $p<0.001$, corrected for multiple comparisons).

\section{Impact of Picture Category, Gender, and Nicotine} Dependence on Rated Dimensions

All images have been divided into 18 categories depending on their content. Separate mixed-design ANOVA revealed a statistically significant interaction between the ratings for all dimensions and picture categories $(F(28.8,393.1)=12.36, p<0.001$, Huynh-Feldt correction). Across all volunteers, pictures depicting persons lighting a cigarette received the highest ratings for urge to

Fig. 1. Distribution of individual and average ratings. a, c, e Histograms of all 3 stimulus dimensions, for all individual ratings. $\mathbf{b}, \mathbf{d}, \mathbf{f}$ The distribution of average ratings over all participants. smoke $(61.23 \pm 24.02)$ and valence $(58.33 \pm 17.16)$. Pictures showing only unlit cigarettes received the highest ratings for arousal $(61.21 \pm 19.40)$. In contrast, pictures depicting cigarette ends received the lowest scores for urge to smoke $(24.80 \pm 21.49)$. This image category also showed the highest discrepancy between urge to smoke $(24.80 \pm 21.49)$ and valence $(36.52 \pm 20.52)$ as well as arousal (44.29 \pm 23.12$)$ ratings. Detailed ratings for each image category are presented in online suppl. Table 2 and Fig. 2. Independent sample $t$ tests showed no statistically significant between-gender differences for urge to smoke (mean $\pm \mathrm{SD}$ : women, $48.15 \pm 9.02$; men, $45.74 \pm 7.67$; $t(37)=0.889, p=0.45$ ), arousal (women, $50.04 \pm 4.91$; men, $49.25 \pm 5.07 ; t(37)=0.493, p=0.76)$, and valence (women, $49.21 \pm 6.03$; men, $48.52 \pm 5.40 ; t(37)=0.41$, $p=0.71$ ). Further, we observed no significant differences in stimuli ratings along the urge to smoke $(t(38)=1.80$, $p=0.91)$, arousal $(t(38)=1.38, p=0.72)$, and valence $(t(38)=1.37, p=0.84)$ dimensions when comparing volunteers with low nicotine dependence according to the Fagerström Test (score of 2 or lower) to volunteers with moderate nicotine dependence (score of 3 or higher). A continuous approach correlating the volunteers' nicotine dependence with their stimulus ratings did not reveal significant results either (urge to smoke: rho $=0.176, p=$ 0.28 ; arousal: rho $=0.022, p=0.89$; valence: $\mathrm{rho}=0.120$, $p=0.46)$. 


\section{Cross-Validation}

For purposes of cross-validation, all volunteers rated 40 additional neutral cues from the IAPS database with respect to arousal and valence as well as 20 neutral and 20 smoking cues from the ISIS database with respect to urge to smoke, arousal, and valence, resulting in 1,600 ratings for the IAPS images as well as 800 ratings for the neutral and smoking cues from the ISIS database, respectively, for each dimension (see online suppl. Table 3). The IAPS stimuli were rated as follows: arousal, mean \pm $\mathrm{SD}=54.15 \pm 20.42$; valence, mean $\pm \mathrm{SD}=52.57 \pm 15.44$. Ratings for arousal and valence were not normally distributed ( $p<0.05$, corrected for multiple comparisons) and significantly correlated with each other ( $r h o=0.497$, $p<0.05)$. Furthermore, arousal ratings differed significantly from the originally reported IAPS scores $(t(39)=8.86, p<0.001)$, while valence ratings did not differ significantly from the originally reported IAPS scores $(t(39)=1.9066, p=0.0603)$. Ratings of the cues selected from the ISIS database were not normally distributed for urge to smoke, arousal, and valence $(p<0.05$, corrected for multiple comparisons). For the neutral ISIS cues, ratings were mean $\pm \mathrm{SD}=38.56 \pm 26.92$ for urge to smoke, mean $\pm \mathrm{SD}=55.75 \pm 19.94$ for arousal, and mean \pm $\mathrm{SD}=52.44 \pm 15.72$ for valence. For the smoking ISIS cues, ratings were as follows: urge to smoke, mean \pm $\mathrm{SD}=61.11 \pm 23.80$; arousal, mean $\pm \mathrm{SD}=62.01 \pm 19.27$; valence, mean $\pm \mathrm{SD}=57.83 \pm 16.31$. Due to lack of reported ratings for neutral ISIS cues, only comparisons for smoking ISIS cues could be performed. They differed significantly between the reported ratings and the newly collected ratings for urge to smoke $(t(19)=-2.22, p=$ $0.04)$ and arousal $(t(19)=19.72, p<0.001)$ but not for valence $(t(19)=1.56, p=0.13)$.

\section{Discussion}

To generate and test a set of individually rated smoking-related images of different intensities along the dimensions craving, valence, and arousal, we collected 250 contemporary smoking-associated pictures from online image repositories. All obtained images were rated by 40 otherwise healthy individuals with TUD with respect to these 3 dimensions. The presented database might contribute to addiction research by enabling more intricate and controlled study designs. Furthermore, the database and the rating tool are available as open-access material in support of the open science initiative, also aiming to improve reproducibility across related studies.

\section{Usability of the SmoCuDa Library}

The novel SmoCuDa database offers 250 images with continuous ratings on a scale from 1 to 100 for the dimensions "urge to smoke," "valence," and "arousal." These 3 dimensions, in particular the "urge to smoke" and "valence" component, are involved in TUD in a different manner [23-25]. Therefore, being able to assess their relative contributions to craving might help gaining a better understanding of relapse [24-28]. Although the 3 dimensions naturally correlated with each other (online suppl. Fig. 2), the database contains also cues that are rated differently on the different dimensions. This SmoCuDa feature can be used for, for example, experiments with parametric designs that disentangle emotional and cravingrelated components of TUD $[29,30]$.

Another feature of the SmoCuDa database is that the ratings for urge to smoke, valence, and arousal are widely distributed along the whole spectrum of the scale (Fig. 1). This complements existing smoking cue datasets, where cues were characterized predominantly by either very high or very low ratings, particularly with respect to the urge to smoke rating [13]. In particular, this wide range of ratings allows for designing experiments with shaping components, for instance, the intensity of presented cues can be varied depending on current craving levels and it is possible to increase difficulty over time. Also, the stimuli cover a wide range of smoking-related scenes depicting the beginning and the end of the smoking process. The consistent difference in rating magnitudes (Fig. 2) is well in line with the current literature, which reported that different stages of the consumption are processed differently on a neuronal level for numerous substances, including nicotine, alcohol, and heroin [31-34]. Therefore, for studies requiring smoking-associated cues that cover the complete range of the urge to smoke, valence, and arousal scale, $\mathrm{SmoCuDa}$ provides a suitable alternative or addition to other databases. Third, using our rating tool, which is published online along with the SmoCuDa database, other researchers will be able to individually assess the urge to smoke, valence, and arousal ratings for each subject. The rating tool can also be useful for extending the database and for including more contemporary images in the future. The SmoCuDa database might also be useful for neuroimaging research, particularly with respect to the potential of cue reactivity for indicating treatment outcome [35-40]. For this research, fMRI-based functional localizers are important to identify subjectspecific brain areas that are activated by craving cues, and $\mathrm{SmoCuDa}$ provides suitable cues for this purpose. Finally, the SmoCuDa images might also serve therapeutic in- 
terventions that require systematically rated craving cues such as exposure therapy or bio-/neurofeedback training $[41,42]$.

\section{Cross-Validations}

Ratings of neutral cues were consistent with previous ratings of these stimuli regarding valence, but arousal ratings differed significantly. This might be because of the different contexts that these images were presented. For instance, the original IAPS study contained quite drastic images, thus providing a more intense affective rating context. This context difference might explain why the original arousal ratings for the IAPS images [16] were lower than in those of the present study. Another reason for cross-study rating differences might have been that we included only individuals with TUD, which was not the case for the other studies. A difference between the samples might also explain why the smoking-related ISIS images were rated higher in urge to smoke by our cohort compared to the original ISIS cohort. Smokers in the current study had an average Fagerström dependence score of 2.7, whereas those in the ISIS study had an average Fagerström dependence score of 1.07.

\section{Limitations}

Our data stem from the analysis of 40 participants. This relatively small sample size might have contributed to the observed heterogeneity of the ratings across participants, as indicated by a rather high standard deviation for some of the scores (online suppl. Table 3). On the other hand, independent sample $t$ tests did not reveal an effect of gender nor of the severity of nicotine dependence on stimulus ratings. The relatively small sample size might have also contributed to the limited cross-validation success. Finally, rating a large amount of stimuli can lead to fatigue and, therefore, weaken the robustness of the ratings [43]. However, even though some subjects reported increased fatigue after rating the 330 images, we observed very high correlations between ratings based on the first 100 presented images and ratings based on all presented images (urge to smoke: rho $=0.880$; arousal: rho $=0.778$; valence: $r h o=0.071$ ), indicating that fatigue did not have a strong influence on stimulus ratings.

\section{Conclusion}

We present a novel image library of contemporary smoking-related cues, which were rated on a continuous scale along the dimensions craving, valence, and arousal.
The cues cover a broad spectrum of ratings along these 3 dimensions. The SmoCuDa database complements and significantly extends existing craving cue databases to allow for more intricate study designs, thus eventually contributing to a better understanding of the neurobiological underpinnings of craving in individuals with TUD.

\section{Acknowledgements}

The authors thank all volunteers for participating in this study.

\section{Statement of Ethics}

This study was conducted in accordance with the Declaration of Helsinki and was approved by the local ethics committee (University of Zurich, Switzerland). Written informed consent was obtained from all participants prior to inclusion in the study.

\section{Disclosure Statement}

All authors declare no conflicts of interest. No support by the industry was received.

\section{Funding Sources}

F.S. was supported by the Swiss National Science Foundation (BSSG10_155915, 100014_178841, 32003B_166566), the Foundation for Research in Science and the Humanities at the University of Zurich (STWF-17-012), and the Baugarten Stiftung. A.M. was supported by the Swiss National Science Foundation (P2SKP3_178107). A.H. was supported by the Forschungskredit of the University of Zurich. M.K. was supported by the National Bank Fellowship Award (Montreal Neurological Institute, McGill University, Canada).

\section{Author Contributions}

F.S., A.M., A.H., and R.S. conceived the project. A.M., A.H., R.S., M.H., B.Q., and F.S. designed the study. L.H., A.B.B., M.K., and E.S. provided resources. A.M. and A.H. collected the data. A.H. and A.M. performed all analyses. A.M., A.H., and F.S. drafted the manuscript. All authors discussed the results and contributed to the final manuscript.

\section{Availability of Data and Material}

The complete SmoCuDa (Smoking Cue Database) library as well as the code for the corresponding rating tool can be downloaded at https://smocuda.github.io. 


\section{References}

1 World Health Organization. WHO report on the global tobacco epidemic, 2017: monitoring tobacco use and prevention policies. World Health Organization; 2017.

2 West R. Tobacco smoking: health impact, prevalence, correlates and interventions. Psychol Health. 2017; 32(8): 1018-36.

3 Fiore MC, Baker TB. Clinical practice. Treating smokers in the health care setting. $\mathrm{N}$ Engl J Med. 2011; 365(13): 1222-31.

4 World Health Organization \& Research for International Tobacco Control. WHO report on the global tobacco epidemic, 2008: the MPOWER package. World Health Organization; 2008.

5 Etter JF, Stapleton JA. Nicotine replacement therapy for long-term smoking cessation: a meta-analysis. Tobacco Control; 2006. 15; p. 280-285. 10.1136/tc.2005.015487.

6 Piasecki TM. Relapse to smoking. Clin Psychol Rev. 2006; 26(2): 196-215.

7 Carter BL, Tiffany ST. Meta-analysis of cue-reactivity in addiction research. Addiction. 1999; 94(3): 327-40.

8 Niaura RS, Rohsenow DJ, Binkoff JA, Monti PM, Pedraza M, Abrams DB. Relevance of cue reactivity to understanding alcohol and smoking relapse. J Abnorm Psychol. 1988; 97(2): 133-52.

9 Engelmann JM, Versace F, Robinson JD, Minnix JA, Lam CY, Cui Y, et al. Neural substrates of smoking cue reactivity: a meta-analysis of fMRI studies. Neuroimage. 2012; 60(1): 25262.

10 Chase HW, Eickhoff SB, Laird AR, Hogarth L. The neural basis of drug stimulus processing and craving: an activation likelihood estimation meta-analysis. Biol Psychiatry. 2011; 70(8): 785-93.

11 Shi Z, Wang AL, Emery LF, Sheerin KM, Romer D. The importance of relevant emotional arousal in the efficacy of pictorial health warnings for cigarettes. Nicotine Tob Res. 2017; 19(6): 750-5.

12 Lubianiker N, Goldway N, Fruchtman-Steinbok T, Paret C, Keynan JN, Singer N, et al. Process-based framework for precise neuromodulation. Nat Hum Behav. 2019; 3(5): 436-45.

13 Gilbert DG, Rabinovich NE. International smoking image series, version1. 1999; 2.

14 Khazaal Y, Zullino D, Billieux J. The Geneva smoking pictures: development and preliminary validation. Eur Addict Res. 2012; 18(3): 103-9.

15 American Psychiatric Association. Diagnostic and statiatical manual of mental disorders $\left(\mathrm{DSM}-5^{\oplus}\right) .5$ th ed. American Psychiatric Pub; 2013.

16 Lang PJ, Bradley MM, Cuthbert BN. International affective picture system (IAPS): technical manual and affective ratings. NIMH Cent Study Emot Atten. 1997; 1: 39-58.

17 Quednow BB, Kühn KU, Hoenig K, Maier W, Wagner M. Prepulse inhibition and habituation of acoustic startle response in male MDMA ('ecstasy') users, cannabis users, and healthy controls. Neuropsychopharmacology. 2004; 29(5): 982-90.

18 Heatherton TF, Kozlowski LT, Frecker RC, Fagerstrom KO. The Fagerstrom test for nicotine dependence: a revision of the Fagerstrom tolerance questionnaire. Br J Addiction. 1991; 86(9): 1119-27. : https: //ai2-s2-pdfs.s3.amaz o n aws.com / 74 c $8 /$ d d 44 c 488807 e 054a5ed8711f6bc7b2fbeaea.pdf.http: //dx.doi. org/10.1111/j.1360-0443.1991.tb01879.x.

19 Etter JF. A self-administered questionnaire to measure cigarette withdrawal symptoms: the cigarette withdrawal scale. Nicotine Tob Res. 2005; 7(1): 47-57.

20 Shiffman S, West RJ, Gilbert DG. Recommendation for the assessment of tobacco craving and withdrawal in smoking cessation trials. Nicotine and Tobacco Research; 2004. 6; p. 599-614. 10.1080/14622200410001734067.

21 Cox LS, Tiffany ST, Christen AG. Evaluation of the brief questionnaire of smoking urges (QSU-brief) in laboratory and clinical settings. Nicotine Tob Res. 2001; 3(1): 7-16. : https: // academic.oup.com/ntr/article-lookup/ doi/10.1080/14622200124218.http: //dx.doi. org/10.1080/14622200020032051.

22 De Cesarei A, Codispoti M. When does size not matter? Effects of stimulus size on affective modulation. Psychophysiology. 2006; 43(2): 207-15.

23 Robinson TE, Berridge KC. The neural basis of drug craving: an incentive-sensitization theory of addiction. Brain Res Brain Res Rev. 1993; 18(3): 247-91

24 Berridge KC, Robinson TE. The mind of an addicted brain: neural sensitization of wanting versus liking. Curr Dir Psychol Sci. 1995; 4(3): 71-5.

25 Berridge KC, Robinson TE. Liking, wanting, and the incentive-sensitization theory of addiction. Am Psychol. 2016; 71(8): 670-9.

26 Robinson MJF, Fischer AM, Ahuja A, Lesser EN, Maniates H. Roles of "wanting" and "liking" in motivating behavior: gambling, food, and drug addictions. Behavioral neuroscience of motivation. Springer; 2015. p. 105-136. 10.1007/7854_2015_387.

27 Wölfling K, Flor H, Grüsser SM. Psychophysiological responses to drug-associated stimuli in chronic heavy cannabis use. Eur J Neurosci. 2008; 27(4): 976-83.

28 Mogg K, Bradley BP, Field M, De Houwer J. Eye movements to smoking-related pictures in smokers: relationship between attentional biases and implicit and explicit measures of stimulus valence. Addiction. 2003; 98(6): 825-36.

29 Amaro E, Barker GJ. Study design in fMRI: basic principles. Brain Cognit. 2006; 60(3): 22032.

30 Dale M. Optimal experimental design for event-related fMRI. Hum Brain Mapp. 1999; 8(2-3): 109-14.

31 Mucha RF, Pauli P, Weber M, Winkler M. Smoking stimuli from the terminal phase of cigarette consumption may not be cues for smoking in healthy smokers. Psychopharmacology. 2008; 201(1): 81-95.

32 Nees F, Diener C, Smolka MN, Flor H. The role of context in the processing of alcohol-relevant cues. Addict Biol. 2012; 17(2): 441-51.

33 Stippekohl B, Winkler M, Mucha RF, Pauli P, Walter B, Vaitl D, et al. Neural responses to begin- and end-stimuli of the smoking ritual in nonsmokers, nondeprived smokers, and deprived smokers. Neuropsychopharmacology. 2010; 35(5): 1209-25.

34 Preller KH, Wagner M, Sulzbach C, Hoenig K, Neubauer J, Franke PE, et al. Sustained incentive value of heroin-related cues in short- and long-term abstinent heroin users. Eur Neuropsychopharmacol. 2013; 23(10): 1270-9.

35 Lee JH, Lim Y, Wiederhold BK, Graham SJ. A functional magnetic resonance imaging (FMRI) study of cue-induced smoking craving in virtual environments. Appl Psychophysiol Biofeedback. 2005; 30(3): 195-204. : http: // www.ncbi.nlm.nih.gov/pubmed/16167185. http: //dx.doi.org/10.1007/s10484-005-6377-z.

36 Goudriaan AE, De Ruiter MB, Van Den Brink W, Oosterlaan J, Veltman DJ. Brain activation patterns associated with cue reactivity and craving in abstinent problem gamblers, heavy smokers and healthy controls: an fMRI study. Addict Biol. 2010; 15(4): 491-503.

37 Li X, Hartwell KJ, Borckardt J, Prisciandaro JJ, Saladin ME, Morgan PS, et al. Volitional reduction of anterior cingulate cortex activity produces decreased cue craving in smoking cessation: a preliminary real-time fMRI study. Addict Biol. 2013; 18(4): 739-48.

38 Franklin TR, Wang Z, Wang J, Sciortino N, Harper D, Li Y, et al. Limbic activation to cigarette smoking cues independent of nicotine withdrawal: a perfusion fMRI study. Neuropsychopharmacology. 2007; 32(11): 2301-9.

39 McBride D, Barrett SP, Kelly JT, Dagher AA, Aw A. Effects of expectancy and abstinence on the neural response to smoking cues in cigarette smokers: an fMRI study. Neuropsychopharmacology. 2006; 31(12): 2728-38.

40 Courtney KE, Schacht JP, Hutchison K, Roche DJ, Ray LA. Neural substrates of cue reactivity: association with treatment outcomes and relapse. Addict Biol. 2016; 21(1): 3-22.

41 Hanlon CA, Hartwell KJ, Canterberry M, Li X, Owens M, LeMatty T, et al. Reduction of cueinduced craving through realtime neurofeedback in nicotine users: the role of region of interest selection and multiple visits. Psychiatry Res. 2013; 213(1): 79-81.

42 Hartwell KJ, Hanlon CA, Li X, Borckardt JJ, Canterberry M, Prisciandaro JJ, et al. Individualized real-time fMRI neurofeedback to attenuate craving in nicotine-dependent smokers. J Psychiatry Neurosci. 2016; 41(1): 48-55.

43 Czajkowski M, Giergiczny M, Greene WH. Learning and fatigue effects revisited: investigating the effects of accounting for unobservable preference and scale heterogeneity. Land Econ. 2015; 90(2): 324-51. 